\title{
The Molecular Era of Brain Tumour Research is upon us: But what now for our Patients?
}

\author{
Timothy Ritzmann, Richard G Grundy, Ruman Rahman*
}

The Children's Brain Tumour Research Centre, School of Medicine, University of Nottingham, UK

"Corresponding author: Ruman Rahman, The Children's Brain Tumour Research Centre, School of Medicine, University of Nottingham, UK, Tel: +0115 82 30993; Fax: +90 256 2120146; E-mail: Ruman.Rahman@nottingham.ac.uk

Received date: Sep 02, 2016; Accepted date: Sep 03, 2016; Published date: Sep 09, 2016

Copyright: ( 2016 Ritzmann T, et al. This is an open-access article distributed under the terms of the Creative Commons Attribution License, which permits unrestricted use, distribution, and reproduction in any medium, provided the original author and source are credited.

\section{Introduction}

There is a natural human desire to seek causation (the intentional stance' described by evolutionary psychologists) or to classify known entities, in order to understand them better. The latter was exemplified by Carl Linnaeus in the 1735, Systema Naturae, but little did he realise however that 280 years later classification systems would have developed so extensively and shifted from a macro to a micro spectrum, that we now use features such as genome-wide genetic and epigenetic profiling, that cannot be observed by the human eye, to delineate disease.

\section{What is the 'Molecular Era' of Brain Tumour Research?}

Historically, brain tumours have been defined by their morphological appearance when examined under the microscope by a pathologist. This has enabled diagnoses of 'ependymoma', 'medulloblastoma' and 'glioblastoma' amongst many other tumour types. With the advent of genomic techniques such as transcriptomic and methylation profiling, researchers are assigning new, 'molecular', diagnoses which are beginning to uncover significant disease heterogeneity even within one morphological tumour type [1]. This editorial asks what the benefits of this approach are and what new challenges might be presented to both researchers and clinicians?

\section{Implications for Research}

The discovery and cladistic description of new tumour subgroups and subtypes within subgroups can make the interpretation of 'presubgroup' research more difficult. Re-analysis of older studies with awareness of the subgroups may allow for fresh biological insights. Findings in one, but not other, subgroups may have been masked by the background noise from other groups - potentially new discoveries could be made from this historical data if the groups can be adequately defined retrospectively.

It is becoming apparent that huge, multi-centre collaborative studies are going to be required to be able to adequately power hypotheses. For example, in 2015, a large multi-cohort study proprosed 9 ependymoma subgroups based on methylation profiling of several hundred tumours [2]. Following this we have seen a move towards classifying some of these subgroups as separate disease entities within the World Health Organisation brain tumour classification, such as the RELA fusion positive supratentorial ependymoma. To be able to adequately study this rare subtype of a rare tumour in the future, extensive collaborative work is going to be required. This will be imperative for both retrospective analysis of tumour cohorts and also for prospectively planned clinical trials. It has also been reported, in an update on Medulloblastoma, that when more samples are included in the research that identifies subgroups, there is a tend towards the discovery of even more subgroups [3], however with the description of 9 ependymoma subgroups outlined above, this also appears to hold true for other tumour types. These approaches have also been particularly revolutionary in the case of Central Nervous System Primitive Neuroectodermal Tumours (CNS-PNET) where four new tumour entities have recently been defined from a previously disparate group of tumours [4]. Further multicentre, collaborative approaches may therefore allow us to define the molecular landscape of these tumour types ever more extensively.

We must also ask ourselves the question, when do we stop defining subgroups? Whilst multiple molecular subgroups are scientifically interesting we must ensure that the findings are also clinically relevant. For example, with respect to outcome or treatment strategies, we must ensure a focus on the translation into clinical practice, where the increasingly accurate definition of these subgroups may allow mechanisms of tumour development to be investigated in greater detail and for the development and testing of more targeted therapies against specific subgroups. We must also be cautious in necessarily regarding a tumour sub-type-specific molecular marker as also representing an amenable therapy target. These markers may be directly associated with a distinct patient group but may not be druggable targets.

\section{Implications for Clinical Practice}

In order for this molecular approach to transform the current clinical setting, the clinicians involved in treating brain tumour patients must be involved and engaged in the debate about how to move these strategies forward and they must also be educated about the developments in this rapidly expanding field in order to be able to apply them for patient benefit. Health organisations and hospitals must keep abreast of the changes to be able to keep up to date with the technology to be able to deliver these advanced molecular tests - in a time of global austerity this may pose a significant challenge, particularly to some health services, such as those in the United Kingdom with an increasingly difficult health economic task.

In a time when access to internet search engines is routine, patients and their families are likely to demand more information about their tumour. The clinicians caring for them will need to understand this information and be able to base appropriate treatment strategies upon it. They will also have to be able to explain when and why these scientific advances aren't ready for transfer into clinical practice, for example, because of a lack of evidence. It could be argued that at the present moment these molecular advances are not making big differences to patients, but time is required to see what true effect they may have in the future. Clinical trials are needed soon to stratify for 
Citation: Ritzmann T, Grundy RG, Rahman R (2016) The Molecular Era of Brain Tumour Research is upon us: But what now for our Patients?. J Brain Tumors Neurooncol 1: e104. doi:10.4172/2475-3203.1000e104

Page 2 of 2

those molecular diagnoses that seem to lend themselves to therapeutic interventions.

Overall, this new molecular era provides exciting opportunities for cancer stratification and management, resulting in genuine potential advancements for patient care, particularly in the design of nextgeneration patient-tailored clinical trials. The caveat to this are that the technologies to deliver these results and the expertise to understand them must be in place across all healthcare systems and this needs to happen from now in order to maximise the potential benefits of these new discoveries. We must also do more work in the scientific community to rapidly validate these findings to ensure that it is safe to transfer them into routine clinical practice.

\section{References}

1. Louis DN, Perry A, Reifenberger G, von Deimling A, Branger DF, et al. (2016) The 2016 World Health Organization Classification of Tumors of the Central Nervous System: a summary. Acta Neuropathol 131: 803-820.

2. Pajtler KW, Witt H, Sill M, Jones DTW, Hovestadt V, et al. (2015) Molecular Classification of Ependymal Tumors across All CNS Compartments, Histopathological Grades, and Age Groups. Canc Cell 27: 728-743.

3. Sturm D, Orr BA, Toprak UH, Hovestadt V, Jones DTW, et al. (2016) New Brain Tumor Entities Emerge from Molecular Classification of CNS-PNETs Resource New Brain Tumor Entities Emerge from Molecular Classification of CNS-PNETs. Cell 164: 1060-1072.

4. Taylor MD, Northcott PA, Korshunov A, Remke M, Cho YJ, et al. (2012) Molecular subgroups of medulloblastoma: the current consensus. Acta neuropathol 123: 465-472. 